\title{
Using cryo-TEM to study the effect of side-chain chemistry on the crystal motifs in polypeptoidnanosheets
}

Morgan Seidler ${ }^{1}$, Nan $\mathrm{Li}^{2}$, Sunting Xuan ${ }^{2}$, David Prendergast ${ }^{2}$, Ronald Zuckermann ${ }^{2}$, Nitash Balsara ${ }^{3}$ and Xi Jiang $^{4}$

${ }^{1}$ Department of Chemical and Biomolecular Engineering, University of California, Berkeley, United States, ${ }^{2}$ Molecular Foundry, Lawrence Berkeley National Laboratory, United States, ${ }^{3}$ University of California, Berkeley, United States, ${ }^{4}$ Materials Sciences Division, Lawrence Berkeley National Laboratory, United States

Polypeptoids are sequence-defined polymers that have received considerable attention as a platform to create biomimetic nanomaterials. Amphiphilic peptoids self-assemble into highly ordered nanosheets in aqueous solution; these nanosheets are held together primarily by interactions between the side chains due to the lack of hydrogen bond donors on the backbone of the molecule [1]. These intermolecular interactions can be finely tuned by varying the chemical structure of the side chains, thereby enabling a systematic study of how the side chain chemistry influences the motifs in the crystal structure, as directly imaged by cryo-TEM $[2,3]$.

The amphiphilic diblockcopolypeptoids are comprised of a hydrophobic block of N-(2- phenylethyl)glycine (Npe) and hydrophilic block of poly(N-2-(2-(2-methoxyethoxy)ethoxy) ethylglycine) (Nte), as illustrated in Figure 1(a). The chlorine atom in Figure 1(a) is highlighted in red because that position (the para-substituent) is varied to hydrogen and bromine in order to study the effect of the side chain chemistry on the self-assembly behavior. Figure 1(b) shows an atomic model of the nanosheets from the projection of the $b$-c plane, wherein the hydrophobic block is crystalline and makes up the core of the nanosheets. Figure 1(c) is a representative TEM image of the dry nanosheets from the direction of the $a-c$ plane.

Low-dose cryo-TEM imaging was employed to limit the radiation damage by the high energy electron beam on the nanosheets. Then a crystal unbending procedure was applied to correct for lattice distortions in the raw micrographs before exporting the images to Relion, which was used to create the averaged atomic-scale image shown in Figure 2(a) [4,5]. This figure is the result of averaging tens of thousands of unit cells, and the bright areas of the image represent the projection views of the glycine backbones along the $b$ direction. The $\mathrm{V}$-shaped less bright areas correspond to the phenyl side chains emanating from the backbones. The neighboring polypeptoids pack into an antiparallel V-shape formation, where the chlorine atoms pack in a tip-to-tip fashion, as seen in Figure 2(a). It is unclear why the chlorine-substituted sheets adopt this antiparallel V-shape formation rather than a parallel $\mathrm{V}$-shape formation as has been previously reported for nanosheets of a similar chemical structure [2].

In order to study the interactions between the side chains on neighboring peptoid monomers and gain further insight into the self-assembly behavior of the nanosheets, molecular dynamics simulations were conducted, and the results of the simulation are represented by the atomic model in Figure 2(b) [6,7]. The image in 2(b) is the same projection from the $a-c$ plane that is shown in the experimental results in Figure 2(a). The MD simulations utilized a dummy atom to model the slight positive potential that occurs on the surface of a covalently-bonded halogen atom. The inclusion of this dummy atom resulted in a simulation that mirrored the experimental results and explained that the electrostatic potential on the surface of the halogen atom is a key factor in determining the crystal motifs and self-assembly behavior.

Small differences in the interactions between neighboring polypeptoid molecules can result in significant changes to the crystal motifs observed in the nanosheets. Understanding the multitude of factors that contribute to the final structure is necessary to synthesize functional nanomaterials with precise control over the physical 
and chemical properties. This goal can only be achieved by collecting high-resolution images of the radiation sensitive polymers in their preserved natural states.

Acknowledgements: Soft Matter Electron Microscopy Program (KC11BN), supported by the Office of Science, Office of Basic Energy Science, US Department of Energy, under Contract DE-AC02-05CH11231. M.S. thanks the National Science Foundation Graduate Research Fellowship Program (Grant number DGE 1752814).

(a)

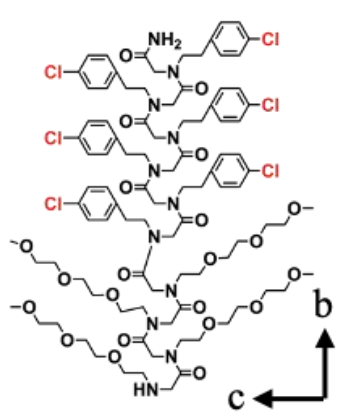

(b)

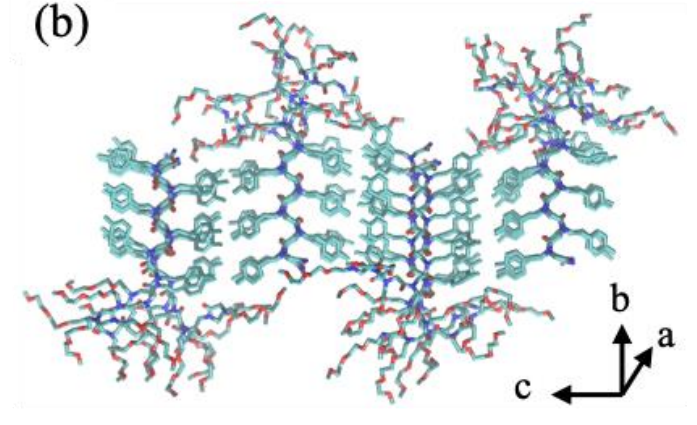

(c)

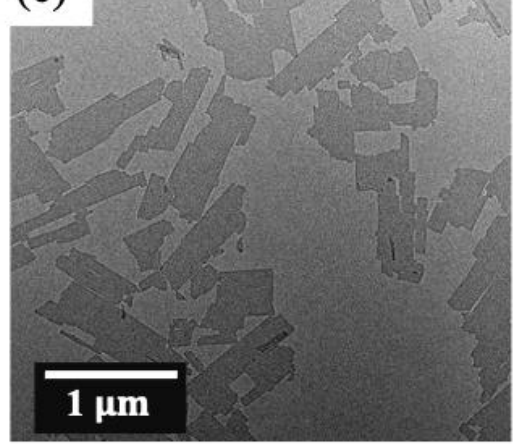

Figure 1. The chemical structure of a Nte4-N4Clpe6 diblockcopolypeptoid molecule (a), a relaxed 3-D atomic model of the self-assembled nanosheets in which the Npe blocks form the ordered core of the nanosheet and the Nte blocks remain amorphous (b), and a bright field TEM image of the Nte4-N4Clpe6 nanosheets from the direction of the a-c plane (c).

(a)

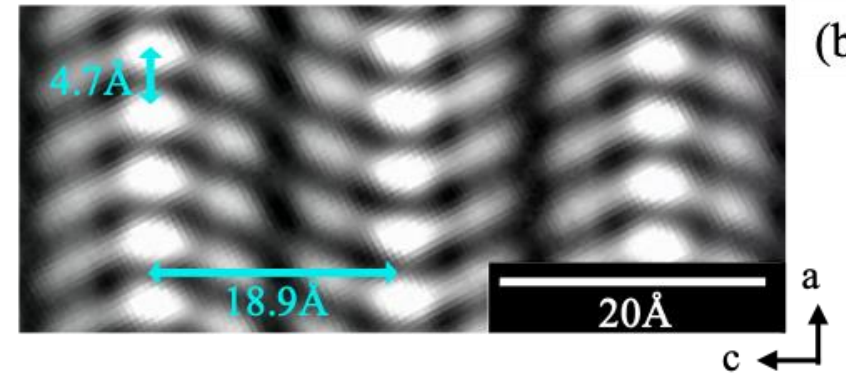

(b)

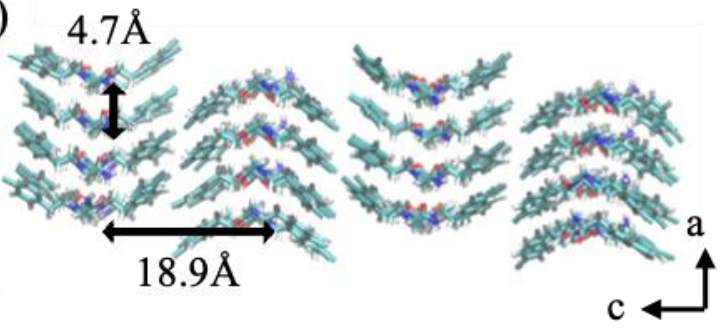

Figure 2. Averaged high resolution image showing the crystal motifs of the Nte4-N4Clpe6 nanosheets from the a-c plane (a). The a and c spacings are labeled on the image. The atomic model shown in (b) was obtained from molecular dynamics simulations, and is represented as the projection through the a-c plane with labels for the a and c spacing of the Nte4-N4Clpe6 sheets. The amorphous Nte blocks are omitted from this view for clarity.

\section{References}

[1] J. Sun and R.N. Zuckermann, ACS Nano 7 (2013), p. 4715.

[2] S. Xuan, et al., PNAS 116 (2019), p. 22491.

[3] X. Jiang, et al., Soft Matter 15 (2019), p. 4723.

[4] B. Gipson, et al., J. Struct. Biol 157 (2007), p. 64.

[5] S.H.W. Scheres, J. Struct. Biol 180 (2012), p. 519.

[6] I. Soteras Gutiérrez, et al., Bioorg. Med. Chem 24 (2016), p. 4812.

[7] L.J. Weiser and E.E. Santiso, J. Comput. Chem 40 (2019), p. 1946. 